\title{
Fine Needle Aspiration Cytology of Pediatric and Adolescent Breast Lesions
}

\author{
Anjali. K. Rao, Parinitha. S. Sangam*, Dinesh Udipi Shastri \\ Department of Pathology, SDM College of Medical Sciences and Hospital, Sattur, Dharwad, India
}

\section{ABSTRACT}

Background: The spectrum of breast lesions in pediatric and adolescent age groups varies distinctly from that of adult breast lesions, in that it is rare and overwhelmingly benign. Histopathological examination being the gold standard in managing these cases, FNAC can be used for the accurate diagnosis in most cases. Aim of the study was to assess spectrum of breast lesions in pediatric and adolescent age groups on fine needle aspiration cytology (FNAC) and to correlate with histopathologic findings.

Methods: This is a 12 year retrospective study of cases during 2004 to 2016.48 patients of age 19 years and below, presenting to our institute with palpable breast lumps were included. FNAC along with histopathologic examination of the excised breast lumps was performed on all the cases.

Result: Majority were females, 46 cases (95.83\%). Most common age group was 16 to 18 years and the most common lesion was fibroadenoma. Other lesions were benign phyllodes, tubular adenoma, hamartoma and gynecomastia. On comparing FNAC with histopathology, concordance was seen in 45 cases $(93.75 \%)$.

Conclusion: In our study all the breast lesions were benign. FNAC and histopathologic examination of these lesions aids in accurate diagnosis and their management.

Keywords: Benign Breast Lesions; Fine Needle Aspiration Cytology; Histopathology; pediatric Breast Lesion.

\section{Introduction}

Breast masses in children and adolescents are rare. The presence of any breast breast lump raises parental concerns of a potential cancer. ${ }^{[1]}$ Majority of breast lesions in this age group have a benign behavior and require only reassurance and observation. ${ }^{[2]} \mathrm{A}$ breast mass in a young boy or girl may arise from abnormal breast development. Most cases of breast enlargement are benign fibroadenoma in girls and Gynecomastia in boys. ${ }^{[3,4]}$ The incidence of breast carcinoma in childhood is less than $0.1 \%$ of all breast cancers and less than $1 \%$ of all childhood cancers. ${ }^{[5,6,7]}$

In the past, excisional biopsy was the only accepted practice, but presently fine needle aspiration cytology (FNAC) makes it possible to reduce unnecessary surgical excision of benign breast lesions. Thus, the present study was planned to evaluate efficacy of FNAC in diagnosing breast lesions and to study the spectrum of breast lesions in children and adolescents.

\section{Materials and Methods}

This was a 12 year retrospective study of cases during Feb 2004 to Jan 2016 carried out in our tertiary care hospital. Both male and female patients of age 19 years and below, who presented with breast lump and who underwent both FNAC and biopsy were included in the study. Patients who did not undergo either FNAC or biopsy were excluded for the study.

After taking informed consent, FNAC of palpable breast lumps were performed using 23 gauge disposable needles attached to $5 \mathrm{ml}$ syringe with a syringe pistol. Slides prepared from the aspirate were stained with $\mathrm{H} \&$ E, Papanicolau and Leishman stains for all cases and examined. Features like cellularity, pattern of cellular arrangement and cell morphology were studied and diagnosis was rendered.

Excision biopsy specimens obtained were grossed and the representative tissue sections were processed using automated tissue processor (Leica 1020) and paraffin blocks prepared. The slides prepared were stained with routine H\&E stain and examined. The histopathology (HP) diagnosis made was correlated with the cytology diagnosis for each case.

The data collected was tabulated, analysed by proportions and percentages, and diagnostic accuracy of test obtained using SPSS software package version 22.

\section{Result}

During the study period 48 patients were studied. 46 were females and two males. Majority of 43 patients $(89.5 \%)$ were between the age of 16 to 19 years followed by 5 
patients $(10.4 \%)$ in 12 to 15 years. 13 patients $(27 \%)$ had multiple breast lumps and 35 patients $(73 \%)$ had solitary breast lumps. Most common location of breast lump was in upper outer quadrant in 24 patients $(35.8 \%)$ followed by upper inner in 16 patients $(23.8 \%)$, lower outer quadrant in $15(22.3 \%)$, lower inner in $7(10.4 \%)$ and retroareolar in 5 cases $(7.4 \%)$. Most of the breast lumps ranged from 2 to $3 \mathrm{~cm}$ in size (47.4\%) followed by 4 to $5 \mathrm{~cm}(18.6 \%)$. The largest lump in one case measured $>10 \mathrm{~cm}$ in size. Two patients $(4.16 \%)$ had rapidly progressive disease, $38(79 \%)$ had gradually progressive disease, and eight $(16.6 \%)$ had non-progressive disease. Majority of the patients had no associated symptoms (66.7\%). Among symptomatic patients, most common symptom was pain in 10 patients (20.8\%) followed by cyclical mastalgia in five $(10.5 \%)$ and bilateral retraction of nipple in one patient (2\%). $93.75 \%$ of breast lumps were firm in consistency, $4.25 \%$ were soft to cystic and $2 \%$ were hard. Two patients had recurrent breast lumps. All the breast lumps were freely mobile except one which showed restricted mobility.

On FNAC, 45 (93.75\%) out of 48 cases were diagnosed as fibroadenomas, two $(4.16 \%)$ as benign breast disease and one $(2.08 \%)$ as gynecomastia. Among 45 diagnosed as fibroadenoma on FNAC 42 were confirmed on histopathology. Two cases were benign phyllodes tumour and one was tubular adenoma on histopathology. Among 2 cases diagnosed as benign breast disease on FNAC one case was fibroadenoma and one was hamartoma on histopathology. One case reported as gynecomastia on FNAC was confirmed on histopathology (Table 1). On correlating FNAC with biopsy 45 out of 48 cases were concordant $(93.75 \%)$. Three cases were discordant $(6.25 \%)$.

FNAC smears from fibroadenoma showed cellular smears with a bimodal pattern containing epithelial and stromal fragments. Large branching sheets of bland epithelial cells with background showing numerous single, bare bipolar nuclei and fragments of fibromyxoid stroma (Fig 1a). Smears from myxoid fibroadenoma showed background containing abundant myxoid material. Excision biopsy from fibroadenomas showed pericanalicular pattern with proliferation of stromal cells around ducts in a circumferential fashion and intracanalicular pattern with compression of the ducts into clefts by the proliferating stromal cells (Fig 1b). Majority of the fibroadenomas diagnosed were of classic type (36 cases). Large myxoid areas were seen in the stroma of myxoid fibroadenoma (one case). One case of cellular fibroadenoma showed highly cellular stroma. One of the fibroadenomas showed large infarcted area on biopsy. One case showed fibroadenoma with marked epitheliosis.

Excision biopsy from tubular adenoma showed wellcircumscribed lesion composed entirely of small round tubules, lined by uniform epithelial cells with surrounding myoepithelial cells and separated by sparse intervening stroma (Fig 2a). This lesion was misdiagnosed as fibroadenoma on FNAC in our study. However, on reviewing the slides few three-dimensional epithelial balls in a background of naked nuclei with no stromal fragments were seen (Fig 2b).

FNAC smears from benign phyllodes which were misdiagnosed as fibroadenoma were reviewed and showed few large cellular stromal fragments. The single stromal cells in the background had retained cytoplasm and appeared plumper than the typical oval bare nuclei seen in fibroadenoma (Fig 3a). The biopsy from the same showed biphasic tumor with increased stromal cellularity and epithelial hyperplasia. The spindle-cell stromal nuclei were monomorphic showing periductal condensation and mitoses $<5$ per 10 high-power fields (Fig 3b).

FNAC from gynecomastia showed sparsely cellular smears with few epithelial cell clusters and small stromal fragments. Histopathology showed ductal epithelial cell hyperplasia with mild stromal edema and fibrosis.

Biopsy from a case of hamartoma showed circumscribed mass with disordered ducts. Few were dilated and showed hyperplasia. Areas of adipose tissue were seen. In this case FNAC was reported as benign breast lesion in view of few benign ductal epithelial cell clusters and adipose tissue fragments.

Table 1: Spectrum of breast lesions on FNAC and biopsy with correlation.

\begin{tabular}{|l|l|l|l|l|l|l|}
\hline \multirow{2}{*}{ FNAC } & \multirow{2}{*}{ No } & HISTOPATHOLOG & Gelign \\
\cline { 3 - 7 } & & Fibroadenoma & $\begin{array}{l}\text { Benign } \\
\text { Phyllodes }\end{array}$ & $\begin{array}{l}\text { Tubular } \\
\text { adenoma }\end{array}$ & Gynecomastia & Hamartoma \\
\hline Fibroadenoma & 45 & 42 & 02 & 01 & --- & --- \\
\hline $\begin{array}{l}\text { Benign breast } \\
\text { disease }\end{array}$ & 02 & 01 & -- & --- & -- & 01 \\
\hline Gynecomastia & 01 & --- & --- & --- & 01 & --- \\
\hline TOTAL & $\mathbf{4 8}$ & $\mathbf{4 3}$ & $\mathbf{0 2}$ & $\mathbf{0 1}$ & $\mathbf{0 1}$ & $\mathbf{0 1}$ \\
\hline
\end{tabular}




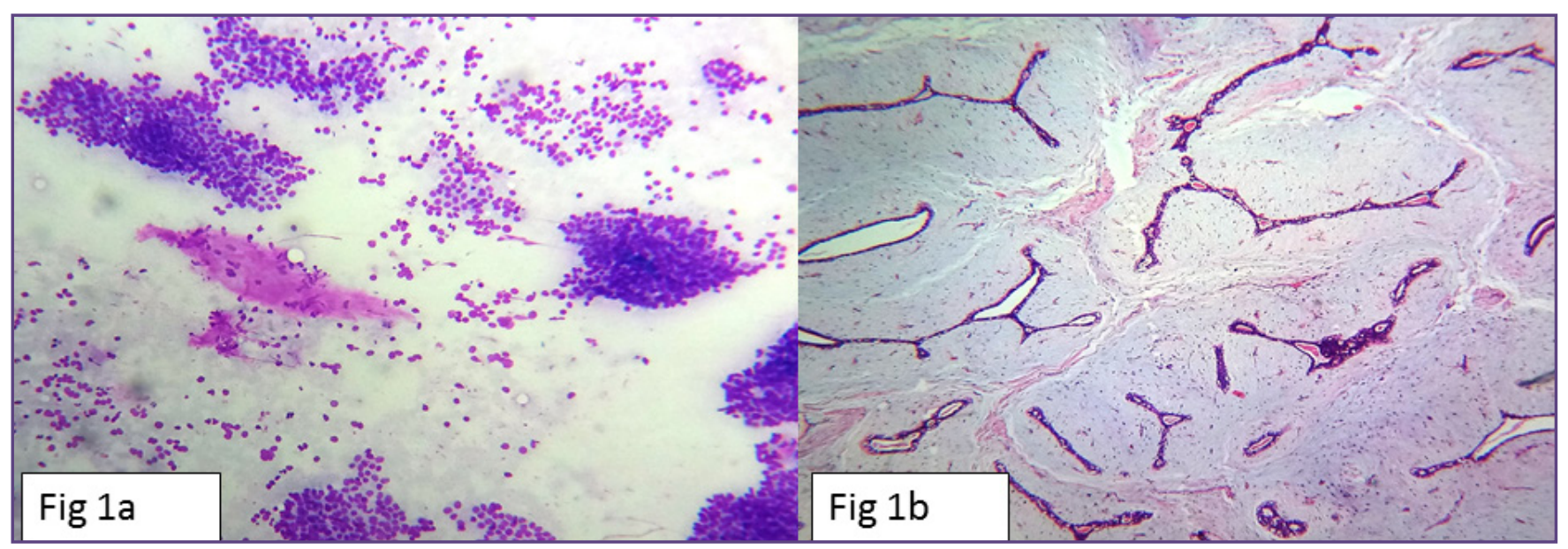

Fig. 1a: FNAC [Leishmans, 40x] Fibroadenoma - Cellular smear with ductal epithelial cell clusters and background of bare bipolar nuclei and stromal fragments. Fig 1b: Histopathology [H\&E, 40x] Fibroadenoma showing compressed ducts surrounded by stroma (Intracanalicular pattern).

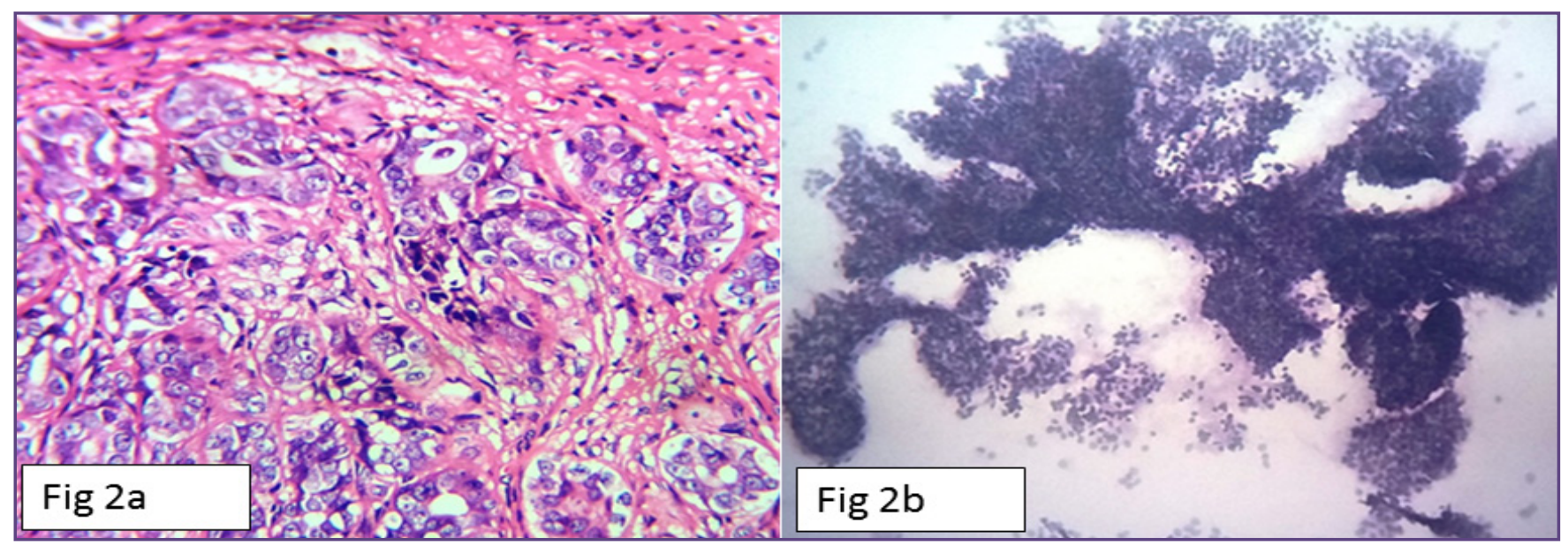

Fig. 2a: Histopathology [H\&E, 400x] Tubular adenoma - Epithelial cells in small tubules and scant stroma. Fig: 2b FNAC [PAP, 400x] Tubular adenoma showing overlapping epithelial cell clusters with few bare nuclei. No stromal fragments seen.

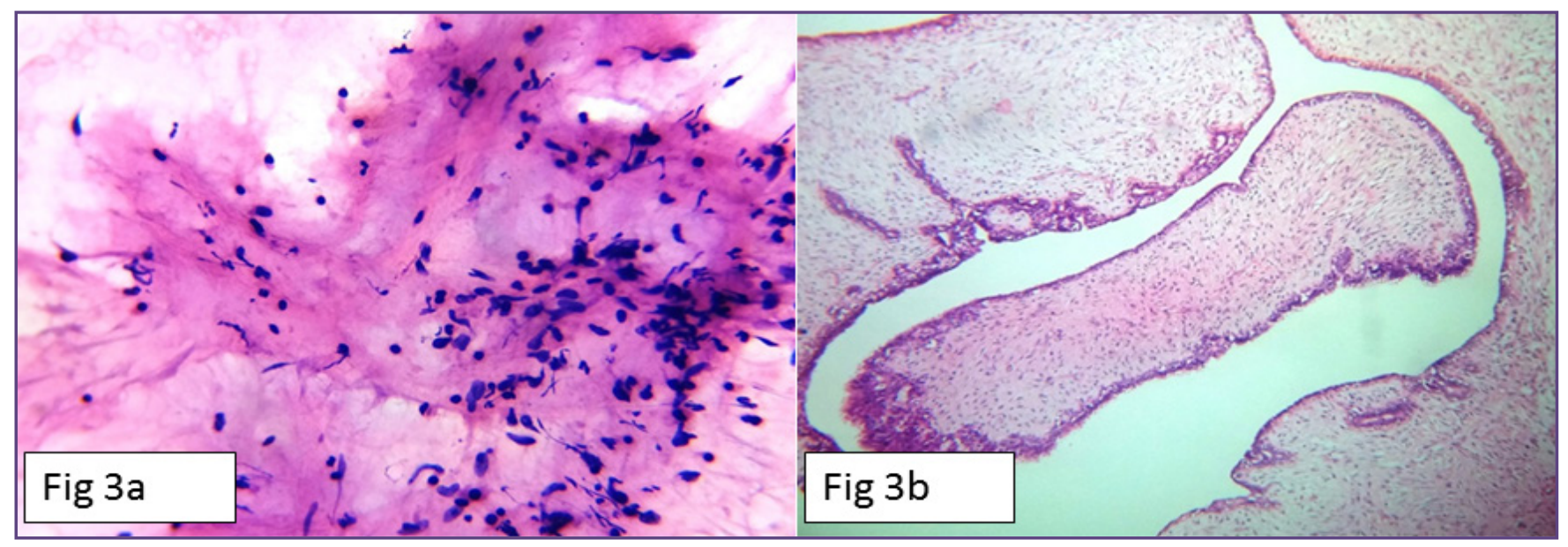

Fig: 3a: FNAC [H\&E, 400x] Benign phyllodes tumour showing cellular stromal fragment Fig: 3b Histopathology [H\&E, 100x] Benign phyllodes - Increased stromal cellularity with periductal condensation and epithelial hyperplasia. 


\section{Discussion}

After onset of puberty, most cases of breast enlargement arise from benign fibroadenoma in girls and Gynecomastia in boys. ${ }^{[2]}$ Other causes of breast masses include infection, trauma, and cyst formation. Hematomas most commonly result from sports or iatrogenic trauma which may present as cystic masses. ${ }^{[3,4]}$ Fibrocystic changes in the breast occur due to physiologic alterations that are very common in the 3 rd decade of life. ${ }^{[3]}$

In the present study most of the breast lumps (89.5\%) were found in age group of 16 to 19 years age which were comparable with other studies like Chung et al (1517 years), Devi et al (11-20 years) and Kapila et al (1621 years). ${ }^{[2,7,8]}$ The most common lesion in our study was fibroadenoma (87.5\%) similar to other studies like Knell et al (93.88\%), Singh et al (89.1\%) and Kaneda et al (91\%). $[9,10,11]$

Majority of the patients in our study had no symptoms. Most common size of fibroadenomas identified ranged between 2 and $3 \mathrm{~cm}$ similar to fibroadenomas identified in other studies like Fallat et al and Zang et al. ${ }^{[1,12]}$ The largest fibroadenoma measured was $10.4 \mathrm{~cm}$ and was diagnosed as a cellular fibroadenoma. Usually fibroadenomas are found as solitary lesions. But 10 to $20 \%$ of conventional fibroadenomas and $25 \%$ of juvenile fibroadenomas may present as multiple or bilateral lumps. 27\% of fibroadenomas were multiple in our study. Fibroadenomas in adolescents unlike in adults can recur at a site adjacent to surgical excision or in contralateral breast. Recurrence of breast lump was seen in 2 cases of our study.

The second most common lesion in our study was benign phyllodes which presented as rapidly growing breast lump with dilated superficial veins. Similarly kapila et al also showed that benign phyllodes tumour was the 2 nd common lesion in this age group. ${ }^{[8]}$

The diagnostic accuracy of FNAC in our study was 93.75\%. Diagnostic accuracy in other studies were Devi et al., 94.7\%, Tiwari $\mathrm{M}$ et al., 90\%, Singh et al., 92.3\% and Chandewala et al., $86.55 \% .^{[7,10,13,14]}$ On FNAC, both cases of benign phyllodes showed more epithelial components and few cellular stromal fragments and thus were misdiagnosed as fibroadenoma in our study. Such fallacies were also seen in Devi et al and also mentioned in literature. ${ }^{[7]}$

FNAC is rapid, inexpensive, minimally invasive procedure which helps in accurate preoperative diagnosis which relieves the patient's anxiety. The definitive treatment can be planned in advance with the informed consent of the patient. Many benign conditions can be confidently diagnosed by FNAC and surgery avoided. The need for frozen section diagnosis is reduced. However, FNAC also has some limitations. A representative sample is required and exact typing of various hyperplastic and low-grade neoplastic lesions may not be possible. When cytology does not correlate with clinical manifestations such as rapid progression or worsening of symptoms a prompt attention by histologic investigation is necessary. ${ }^{[7]}$

\section{Conclusion}

In our study all breast lesions in children and adolescents were benign. Most common lesion was fibroadenoma. FNAC can accurately diagnose these lesions in majority of the cases with breast lumps and is the initial tool for diagnosis. When cytology does not correlate with clinical symptoms histopathologic studies are necessary.

\section{Acknowledgements}

Department of surgery

\section{Reference}

1. Fallat $\mathrm{M}$, Ignacio R. Breast Disorders in Children and Adolescents. Journal of Pediatric and Adolescent Gynecology 2008; 21(6):311-16.

2. Chung E, Cube R, Hall G, González C, Stocker J, Glassman L. Breast Masses in Children and Adolescents: Radiologic-Pathologic Correlation. RadioGraphics 2009; 29(3):907-31.

3. Greydanus DE, Matytsina L, Gains M. Breast disorders in children and adolescents. Prim Care 2006; 33:455-502.

4. Siegel MJ. Chest. In: Siegel MJ, ed. Pediatric sonography. 3rd ed. Philadelphia, Pa: Lippincott Williams \& Williams, 2002; 201-211.

5. Shannon C, Smith IE. Breast cancer in adolescents and young women. Eur J Cancer 2003; 39:2632-42.

6. Tea M-K, Asseryanis E, Kroiss R, et al. Surgical breast lesions in adolescent females. Pediatr Surg Int 2009; 25:73-75.

7. Devi G, Datta B, Medhi K, Goswami R, Baruah D. Pattern of Benign Breast Lesions on Fine Needle Aspiration Cytology. Indian journal of research 2016;5(5):484-87.

8. Kapila K, Pathan S, Al-Mosawy F, George S, Haji B, AlAyadhy B. Fine Needle Aspiration Cytology of Breast Masses in Children and Adolescents. Acta Cytologica 2008;52(6):681-86.

9. Knell J, Koning J, Grabowski J. Analysis of surgically excised breast masses in 119 pediatric patients. Pediatr Surg Int 2015; 32(1):93-96.

10. Singh A, Haritwal A and Murali BM. Pattern of Breast Lumps and Diagnostic Accuracy of Fine Needle Aspiration Cytology: A Hospital Based Study From Pondicherry, India. The Internet J of Pathol 2011; 11(2):1-6. 
11. Kaneda H, Mack J, Kasales C, Schetter S. Pediatric and Adolescent Breast Masses: A Review of Pathophysiology, Imaging, Diagnosis, and Treatment. American Journal of Roentgenology 2013; 200(2):204-12.

12. Zhang R, Bevan S, Sun P, Jim Z Lu, Peng. Unusual Presentation of Multiple Fibroadenomas in Bilateral Breasts and Axillary Accessory Breast. Breast cancer: basic and clinical research 2012; 6(1):95-99.
13. Tiwari M. Role of fine needle aspiration cytology in diagnosis of breast lumps. Kathmandu University Medical Journal 2007; 5(2): 215-17.

14. Chandanwale, S Rajpal M, Jadhav P, Sood S, Gupta K , Gupta N ".Pattern of benign breast lesions on FNAC in consecutive 100 cases, Int J Pharm Bio Sci 2013;3(4):129138.

*Corresponding author:

Dr. Parinitha. S. Sangam, Associate professor pathology. SDM College of Medical sciences and Hospital, Sattur. Dharwad-580009. Karnataka state. India Phone: +91 9481217924

Email: drparinithabhat@gmail.com

Financial or other Competing Interests: None. 